\title{
MAPPINGS AND DECOMPOSITIONS OF CONTINUITY ON ALMOST LINDELÖF SPACES
}

\author{
A. J. FAWAKHREH AND A. KILIÇMAN \\ Received 18 September 2005; Revised 4 January 2006; Accepted 12 March 2006
}

A topological space $X$ is said to be almost Lindelöf if for every open cover $\left\{U_{\alpha}: \alpha \in \Delta\right\}$ of $X$ there exists a countable subset $\left\{\alpha_{n}: n \in \mathbb{N}\right\} \subseteq \Delta$ such that $X=\bigcup_{n \in \mathbb{N}} \operatorname{Cl}\left(U_{\alpha_{n}}\right)$. In this paper we study the effect of mappings and some decompositions of continuity on almost Lindelöf spaces. The main result is that a $\theta$-continuous image of an almost Lindelöf space is almost Lindelöf.

Copyright (c) 2006 Hindawi Publishing Corporation. All rights reserved.

\section{Introduction}

Among the various covering properties of topological spaces a lot of attention has been given to those covers which involve open and regularly open sets. In 1982 Balasubramanian [4] introduced and studied the notion of nearly Lindelöf spaces and in 1984 Willard and Dissanayake [21] gave the notion of almost Lindelöf spaces. Then in 1996 Cammaroto and Santoro [5] studied and gave further new results about these spaces which are considered as one of the main generalizations of Lindelöf spaces.

Moreover, decompositions of continuity have been recently of major interest among general topologists. They are being studied by many authors, including Singal and Singal [19], Popa and Stan [16], Noiri [14], Long and Herrington [11], Mashhour et al. [12], Abd El-Monsef et al. [1], Dontchev [6], Dontchev and Przemski [7], Nasef and Noiri [13], Park and Ha [15], and Baker [2, 3]. In fact, mathematicians introduced in several papers different and interesting new decompositions of continuity as well as generalized continuous functions.

Throughout the present paper, spaces always mean topological spaces on which no separation axioms are assumed unless explicitly stated. The topological space $(X, \tau)$ will be replaced by $X$ if there is no chance for confusion. The interior and the closure of any subset $A$ of $(X, \tau)$ will be denoted by $\operatorname{Int}(A)$ and $\mathrm{Cl}(A)$, respectively.

The purpose of this paper is to study the effect of mappings and some decompositions of continuity on almost Lindelöf spaces. We also show that some mappings preserve this property. The main result is that the image of an almost Lindelöf space under a $\theta$-continuous function is almost Lindelöf. 


\section{Preliminaries}

Recall that a subset $A \subseteq X$ is called regularly open (regularly closed) if $A=\operatorname{Int}(\mathrm{Cl}(A))$ $(A=\mathrm{Cl}(\operatorname{Int}(A)))$. The topology generated by the regularly open subsets of a space $(X, \tau)$ is called the semiregularization of $(X, \tau)$ and is denoted by $\left(X, \tau_{s}\right)$. A space $(X, \tau)$ is said to be semiregular if the regularly open sets form a base for the topology or equivalently $\tau=\tau_{s}$. It is called almost regular if for any regularly closed set $C$ and any singleton $\{x\}$ disjoint from $C$, there exist two disjoint open sets $U$ and $V$ such that $C \subseteq U$ and $x \in V$. Note that a space $X$ is regular if and only if it is semiregular and almost regular [17]. Moreover, a space $X$ is said to be submaximal if every dense subset of $X$ is open in $X$ and it is called extremally disconnected if the closure of each open set of $X$ is open in $X$. A space $X$ is said to be mildly normal [20] if whenever $A$ and $B$ are disjoint regularly closed sets in $X$, then there are two disjoint open sets $U$ and $V$ with $A \subseteq U$ and $B \subseteq V$. And it is called nearly paracompact [18] if every regularly open cover of $X$ admits an open locally finite refinement.

Definition 2.1 (see [4]). A topological space $X$ is said to be nearly Lindelöf if for every open cover $\left\{U_{\alpha}: \alpha \in \Delta\right\}$ of $X$, there exists a countable subset $\left\{\alpha_{n}: n \in \mathbb{N}\right\} \subseteq \Delta$ such that $X=\bigcup_{n \in \mathbb{N}} \operatorname{Int}\left(\mathrm{Cl}\left(U_{\alpha_{n}}\right)\right)$. That is, every regularly open cover of $X$ admits a countable subcover.

Mappings and decompositions of continuity on nearly Lindelöf spaces were recently studied by the authors in [8].

Definition 2.2. Let $(X, \tau)$ and $(Y, \sigma)$ be topological spaces. A function $f: X \rightarrow Y$ is said to be

(1) almost continuous [19] if $f^{-1}(V)$ is open in $X$ for every regularly open set $V$ in $Y$;

(2) precontinuous [12] (resp., $\beta$-continuous [1]) if $f^{-1}(V) \subseteq \operatorname{Int}\left(\operatorname{Cl}\left(f^{-1}(V)\right)\right.$ ) (resp., $\left.f^{-1}(V) \subseteq \mathrm{Cl}\left(\operatorname{Int}\left(\mathrm{Cl}\left(f^{-1}(V)\right)\right)\right)\right)$ for every open set $V$ in $Y$;

(3) almost precontinuous (resp., almost $\beta$-continuous) [13] if for each $x \in X$ and each regularly open set $V$ in $Y$ containing $f(x)$, there exists a set $U$ in $X$ containing $x$ with $U \subseteq \operatorname{Int}(\mathrm{Cl}(U))$ (resp., $U \subseteq \mathrm{Cl}(\operatorname{Int}(\mathrm{Cl}(U)))$ ) such that $f(U) \subseteq V$;

(4) $\delta$-continuous [14] (resp., almost $\delta$-continuous) if for each $x \in X$ and each regularly open set $V$ of $Y$ containing $f(x)$, there exists a regularly open set $U$ of $X$ containing $x$ such that $f(U) \subseteq V$ (resp., $f(U) \subseteq \mathrm{Cl}(V)$ );

(5) $\theta$-continuous [9] (resp., strong $\theta$-continuous [11]) if for every $x \in X$ and every open subset $V$ of $Y$ containing $f(x)$, there exists an open subset $U$ in $X$ containing $x$ such that $f(\mathrm{Cl}(U)) \subseteq \mathrm{Cl}(V)$ (resp., $f(\mathrm{Cl}(U)) \subseteq V)$;

(6) weakly quasicontinuous [16] if for each $x \in X$, each open set $G$ of $X$ containing $x$ and each open set $V$ of $Y$ containing $f(x)$, there exists an open set $U$ of $X$ such that $\phi \neq U \subseteq G$ and $f(U) \subseteq \mathrm{Cl}(V)$;

(7) contra-continuous [6] if $f^{-1}(V)$ is closed in $X$ for every open set $V$ in $Y$;

(8) subcontra-continuous [3] if there exists an open base $\mathscr{B}$ for the topology on $Y$ such that $f^{-1}(V)$ is closed in $X$ for every $V \in \mathscr{B}$.

Note that almost $\delta$-continuity is equivalent to weakly $\delta$-continuity due to Baker [2]. Also, in [8], the following diagram in which none of its implications is reversible, is given. 
It illustrates the relations among some of these mappings:

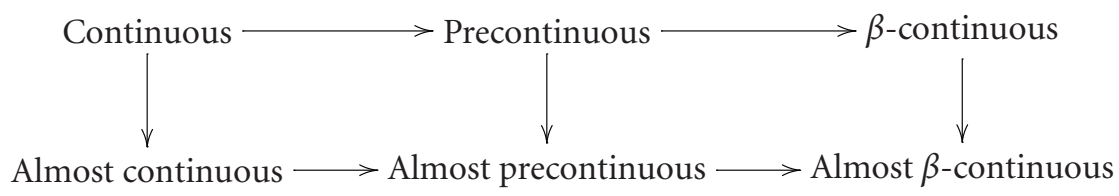

Moreover, it is clear that every strong $\theta$-continuous mapping is $\theta$-continuous and $\delta$ continuous. It is clear also, that every $\delta$-continuous mapping is almost $\delta$-continuous but the converse is not true as Example 3.5 below shows. Contra-continuity implies subconracontinuity but the converse, in general, is not true (see [3]). So, with Lemma 3.12 and Example 3.13 below, we obtain the following diagram in which none of these implications is reversible:

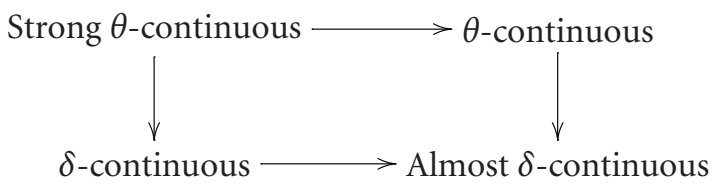

\section{Almost Lindelöf spaces}

Definition 3.1 (see [21]). A topological space $X$ is said to be almost Lindelöf if for every open cover $\left\{U_{\alpha}: \alpha \in \Delta\right\}$ of $X$ there exists a countable subset $\left\{\alpha_{n}: n \in \mathbb{N}\right\} \subseteq \Delta$ such that $X=\bigcup_{n \in \mathbb{N}} \operatorname{Cl}\left(U_{\alpha_{n}}\right)$.

Note that every Lindelöf space is nearly Lindelöf and every nearly Lindelöf space is almost Lindelöf but the converses, in general, are not true (see [5]). Moreover, it is well known that the continuous image of a Lindelöf space is Lindelöf and in [8] it was shown that the $\delta$-continuous image of a nearly Lindelöf space is nearly Lindelöf. In the case of almost Lindelöf spaces we give the following theorem.

Theorem 3.2. Let $f: X \rightarrow Y$ be a $\theta$-continuous surjection from $X$ into $Y$. If $X$ is almost Lindelöf, then $Y$ is almost Lindelöf.

Proof. Let $\left\{V_{\alpha}: \alpha \in \Delta\right\}$ be an open cover of $Y$. Let $x \in X$ and $V_{\alpha_{x}}$ be an open set in $Y$ such that $f(x) \in V_{\alpha_{x}}$. Since $f$ is $\theta$-continuous, there exists an open set $U_{\alpha_{x}}$ of $X$ containing $x$ such that $f\left(\mathrm{Cl}\left(U_{\alpha_{x}}\right)\right) \subseteq \mathrm{Cl}\left(V_{\alpha_{x}}\right)$. Now $\left\{U_{\alpha_{x}}: x \in X\right\}$ is an open cover of the almost Lindelöf space $X$. So there exists a countable subset $\left\{U_{\alpha_{x_{n}}}: n \in \mathbb{N}\right\}$ such that $X=\bigcup_{n \in \mathbb{N}} \operatorname{Cl}\left(U_{\alpha_{x_{n}}}\right)$. Thus

$$
Y=f(X)=f\left(\bigcup_{n \in \mathbb{N}} \mathrm{Cl}\left(U_{\alpha_{x_{n}}}\right)\right)=\bigcup_{n \in \mathbb{N}} f\left(\operatorname{Cl}\left(U_{\alpha_{x_{n}}}\right)\right) \subseteq \bigcup_{n \in \mathbb{N}} \operatorname{Cl}\left(V_{\alpha_{x_{n}}}\right) .
$$

This implies that $Y$ is almost Lindelöf and completes the proof.

Corollary 3.3. The $\theta$-continuous image of an almost Lindelöf space is almost Lindelöf.

Lemma 3.4 (see [10]). If $f:(X, \tau) \rightarrow(Y, \sigma)$ is almost continuous, then $f$ is $\theta$-continuous. 
The converse of Lemma 3.4 is not true, in general, as the following example shows.

Example 3.5. Let $X=\{a, b, c\}$. Define on $X$ the following two topologies $\tau=\{\phi$, $\{b\},\{b, c\}, X\}$ and $\sigma=\{\phi,\{a\},\{b\},\{a, b\}, X\}$. Let $f:(X, \tau) \rightarrow(X, \sigma)$ be a function defined as follows: $f(a)=b$ and $f(b)=f(c)=c$. Then $f$ is $\theta$-continuous but it is not almost continuous since $f^{-1}(\{b\})=\{a\}$ is not open in $(X, \tau)$ whereas $\{b\}$ is regularly open in $(X, \sigma)$. Since $f$ is $\theta$-continuous, by Lemma 3.12 below, $f$ is almost $\delta$-continuous but it is not $\delta$-continuous since, there is a regularly open set $\{b\}$ of $Y$ containing $f(\{a\})$ but there is no regularly open set $U$ of $X$ containing $\{a\}$ such that $f(U) \subseteq\{b\}$.

Corollary 3.6. Let $f: X \rightarrow Y$ be an almost continuous surjection from $X$ into $Y$. If $X$ is almost Lindelöf, then $Y$ is almost Lindelöf.

Since every continuous function is almost continuous and by Lemma 3.4, we conclude the following corollary.

COROLlary 3.7. Almost Lindelöf property is a topological property.

Note also that an almost regular space is almost Lindelöf if and only if it is nearly Lindelöf (see [4]). Moreover, an almost regular and nearly Lindelöf space is nearly paracompact and mildly normal (see [5]). Thus we have the following corollary by using Theorem 3.2.

Corollary 3.8. Let $f: X \rightarrow Y$ be $\theta$-continuous surjection from $X$ into an almost regular space $Y$. If $X$ is almost Lindelöf, then $Y$ is nearly Lindelöf, mildly normal, and nearly paracompact.

Proposition 3.9. Let $f: X \rightarrow Y$ be strong $\theta$-continuous surjection from $X$ into $Y$. If $X$ is almost Lindelöf, then $Y$ is Lindelöf.

Proof. Let $\left\{V_{\alpha}: \alpha \in \Delta\right\}$ be an open cover of $Y$. Let $x \in X$ and $V_{\alpha_{x}}$ be an open set in $Y$ such that $f(x) \in V_{\alpha_{x}}$. Since $f$ is strong $\theta$-continuous, there exists an open set $U_{\alpha_{x}}$ of $X$ containing $x$ such that $f\left(\mathrm{Cl}\left(U_{\alpha_{x}}\right)\right) \subseteq V_{\alpha_{x}}$. Now $\left\{U_{\alpha_{x}}: x \in X\right\}$ is an open cover of the almost Lindelöf space $X$. So there exists a countable subset $\left\{U_{\alpha_{x_{n}}}: n \in \mathbb{N}\right\}$ such that $X=\bigcup_{n \in \mathbb{N}} \operatorname{Cl}\left(U_{\alpha_{x_{n}}}\right)$. Thus

$$
Y=f(X)=f\left(\bigcup_{n \in \mathbb{N}} \mathrm{Cl}\left(U_{\alpha_{x_{n}}}\right)\right)=\bigcup_{n \in \mathbb{N}} f\left(\operatorname{Cl}\left(U_{\alpha_{x_{n}}}\right)\right) \subseteq \bigcup_{n \in \mathbb{N}} V_{\alpha_{x_{n}}} .
$$

This implies that $Y$ is Lindelöf and completes the proof.

Corollary 3.10. The strong $\theta$-continuous image of an almost Lindelöf space is Lindelöf.

Note that if $f:(X, \tau) \rightarrow(Y, \sigma)$ is a strong $\theta$-continuous function, then $f$ is continuous but the converse is not true, in general, as in Example 3.5.

Next we prove that almost $\delta$-continuous image of a nearly Lindelöf space is almost Lindelöf.

Proposition 3.11. Let $f: X \rightarrow Y$ be an almost $\delta$-continuous surjection from $X$ into $Y$. If $X$ is nearly Lindelöf, then $Y$ is almost Lindelöf. 
Proof. Let $\mathscr{V}=\left\{V_{\alpha}: \alpha \in \Delta\right\}$ be an open cover of $Y$. Let $x \in X$ and $V_{\alpha_{x}} \in \mathscr{V}$ such that $f(x) \in V_{\alpha_{x}} \subseteq \operatorname{Int}\left(\mathrm{Cl}\left(V_{\alpha_{x}}\right)\right)$. Since $f$ is almost $\delta$-continuous and $\operatorname{Int}\left(\mathrm{Cl}\left(V_{\alpha_{x}}\right)\right)$ is regularly open in $(Y, \sigma)$ containing $f(x)$, then for every $x \in X$, there exists a regularly open subset $U_{\alpha_{x}}$ of $X$ containing $x$ such that $f\left(U_{\alpha_{x}}\right) \subseteq \operatorname{Cl}\left(V_{\alpha_{x}}\right)$. So $\left\{U_{\alpha_{x}}: x \in X\right\}$ is a regularly open cover of the nearly Lindelöf space $X$. Thus there exists a countable subset $\left\{x_{n}: n \in N\right\}$ such that $X=\bigcup_{n \in \mathbb{N}} U_{\alpha_{x_{n}}}$. So

$$
Y=f(X)=f\left(\bigcup_{n \in \mathbb{N}} U_{\alpha_{x_{n}}}\right)=\bigcup_{n \in \mathbb{N}} f\left(U_{\alpha_{x_{n}}}\right) \subseteq \bigcup_{n \in \mathbb{N}} \mathrm{Cl}\left(V_{\alpha_{x_{n}}}\right) .
$$

This shows that $Y$ is almost Lindelöf.

Now we prove that $\theta$-continuity implies almost $\delta$-continuity.

Lemma 3.12. If $f:(X, \tau) \rightarrow(Y, \sigma)$ is $\theta$-continuous, then $f$ is almost $\delta$-continuous.

Proof. Let $x \in X$ and $V$ be a regularly open subset of $Y$ containing $f(x)$. Since $f$ is $\theta$ continuous, there exists an open subset $U$ of $X$ containing $x$ such that $f(\mathrm{Cl}(U)) \subseteq \mathrm{Cl}(V)$. Thus $U \subseteq \operatorname{Int}(\mathrm{Cl}(U))$ and $\operatorname{Int}(\mathrm{Cl}(U))$ is a regularly open subset of $X$ containing $x$ such that $f(\operatorname{Int}(\mathrm{Cl}(U))) \subseteq f(\mathrm{Cl}(U)) \subseteq \mathrm{Cl}(V)$. This implies that $f$ is almost $\delta$-continuous and completes the proof.

The converse of Lemma 3.12 is not true, in general, as the following example shows.

Example 3.13. Let $X=\{a, b, c, d\}$ and $Y=\{x, y, z\}$. Then we define the topologies $\tau=$ $\{\phi,\{c\},\{d\},\{a, c\},\{c, d\},\{a, c, d\}, X\}, \sigma=\{\phi,\{x\},\{y\},\{x, y\}, Y\}$ on $X, Y$, respectively. Now if $f:(X, \tau) \rightarrow(Y, \sigma)$ is a function defined as $f(a)=z$ and $f(b)=f(c)=f(d)=y$, then $f$ is almost $\delta$-continuous but it is not $\theta$-continuous.

Next we prove the following proposition.

Proposition 3.14. Let $f: X \rightarrow Y$ be a weakly quasicontinuous and precontinuous surjection from $X$ into $Y$. If $X$ is nearly Lindelöf, then $Y$ is almost Lindelöf.

Proof. Let $\left\{V_{\alpha}: \alpha \in \Delta\right\}$ be an open cover of $Y$. So $\left\{f^{-1}\left(V_{\alpha}\right): \alpha \in \Delta\right\}$ is a cover of $X$. Since $f$ is precontinuous, $f^{-1}\left(V_{\alpha}\right) \subseteq \operatorname{Int}\left(\mathrm{Cl}\left(f^{-1}\left(V_{\alpha}\right)\right)\right)$. So $\left\{\operatorname{Int}\left(\mathrm{Cl}\left(f^{-1}\left(V_{\alpha}\right)\right)\right): \alpha \in \Delta\right\}$ is a regularly open cover of the nearly Lindelöf space $X$. It follows that there exists a countable subset $\left\{\alpha_{n}: n \in \mathbb{N}\right\} \subseteq \Delta$ such that $X=\bigcup_{n \in \mathbb{N}} \operatorname{Int}\left(\operatorname{Cl}\left(f^{-1}\left(V_{\alpha_{n}}\right)\right)\right)$. Since $f$ is weakly quasicontinuous, $\operatorname{Int}\left(\mathrm{Cl}\left(f^{-1}\left(V_{\alpha_{n}}\right)\right)\right) \subseteq f^{-1}\left(\mathrm{Cl}\left(V_{\alpha_{n}}\right)\right)$ (see [15, Theorem 3.3]). So $X=$ $\bigcup_{n \in \mathbb{N}} f^{-1}\left(\mathrm{Cl}\left(V_{\alpha_{n}}\right)\right)$. Thus

$$
Y=f(X)=f\left(\bigcup_{n \in \mathbb{N}} f^{-1}\left(\mathrm{Cl}\left(V_{\alpha_{n}}\right)\right)\right)=\bigcup_{n \in \mathbb{N}} f\left(f^{-1}\left(\mathrm{Cl}\left(V_{\alpha_{n}}\right)\right)\right) \subseteq \bigcup_{n \in \mathbb{N}} \mathrm{Cl}\left(V_{\alpha_{n}}\right) .
$$

This implies that $Y$ is almost Lindelöf.

We also prove the following proposition.

Proposition 3.15. The image of an almost Lindelöf space under a precontinuous and subcontra-continuous mapping is Lindelöf. 
Proof. Let $f: X \rightarrow Y$ be a subcontra-continuous and precontinuous function from $X$ to $Y$. Assume that $X$ is almost Lindelöf. Let $\mathscr{B}$ be an open base for the topology on $Y$ for which $f^{-1}(V)$ is closed in $X$ for every $V \in \mathscr{B}$. Let $\mathcal{U}=\left\{U_{\alpha}: \alpha \in \Delta\right\}$ be an open cover of $f(X)$. For each $x \in X$, let $U_{\alpha_{x}} \in \mathcal{U}$ such that $f(x) \in U_{\alpha_{x}}$. Then there exists $V_{\alpha_{x}} \in \mathscr{B}$ such that $f(x) \in V_{\alpha_{x}} \subseteq U_{\alpha_{x}}$. Since $f$ is subcontra-continuous, $f^{-1}\left(V_{\alpha_{x}}\right)$ is closed in $X$. Since $f$ is precontinuous, $f^{-1}\left(V_{\alpha_{x}}\right) \subseteq \operatorname{Int}\left(\mathrm{Cl}\left(f^{-1}\left(V_{\alpha_{x}}\right)\right)\right)=\operatorname{Int}\left(f^{-1}\left(V_{\alpha_{x}}\right)\right)$. So $f^{-1}\left(V_{\alpha_{x}}\right)=$ $\operatorname{Int}\left(f^{-1}\left(V_{\alpha_{x}}\right)\right)$. It follows that $f^{-1}\left(V_{\alpha_{x}}\right)$ is clopen and hence $\left\{f^{-1}\left(V_{\alpha_{x}}\right): x \in X\right\}$ is a clopen cover of the almost Lindelöf space $X$. So there exists a countable subfamily $\left\{x_{n}: n \in \mathbb{N}\right\}$ for which

$$
X=\bigcup_{n \in \mathbb{N}} \operatorname{Cl}\left(f^{-1}\left(V_{\alpha_{x_{n}}}\right)\right)=\bigcup_{n \in \mathbb{N}} f^{-1}\left(V_{\alpha_{x_{n}}}\right) \subseteq \bigcup_{n \in \mathbb{N}} f^{-1}\left(U_{\alpha_{x_{n}}}\right)
$$

Thus we have

$$
f(X)=f\left(\bigcup_{n \in \mathbb{N}} f^{-1}\left(U_{\alpha_{x_{n}}}\right)\right)=\bigcup_{n \in \mathbb{N}} f\left(f^{-1}\left(U_{\alpha_{x_{n}}}\right)\right) \subseteq \bigcup_{n \in \mathbb{N}} U_{\alpha_{x_{n}}} .
$$

This implies that $f(X)$ is Lindelöf and completes the proof.

Since contra-continuous functions are subcontra-continuous, we have the following corollary.

Corollary 3.16. The image of an almost Lindelöf space under a contra-continuous and precontinuous mapping is Lindelöf.

The following two propositions are analogous results of nearly Lindelöf spaces (see $[8])$.

Proposition 3.17. Let $f: X \rightarrow Y$ be an almost $\beta$-continuous surjection. If $X$ is submaximal, extremally disconnected, and almost Lindelöf, then $Y$ is almost Lindelöf.

Proof. The proof follows immediately from [13, Theorem 4.3] and Corollary 3.6.

Proposition 3.18. Let $f: X \rightarrow Y$ be an almost precontinuous surjection. If $X$ is submaximal and almost Lindelöf, then $Y$ is almost Lindelöf.

Proof. The proof follows immediately from [13, Theorem 4.4] and Corollary 3.6.

\section{References}

[1] M. E. Abd El-Monsef, S. N. El-Deeb, and R. A. Mahmoud, $\beta$-open sets and $\beta$-continuous mapping, Bulletin of the Faculty of Science. Assiut University. A. Physics and Mathematics 12 (1983), no. 1, 77-90.

[2] C. W. Baker, On super continuous functions, Bulletin of the Korean Mathematical Society 22 (1985), no. 1, 17-22.

[3] Subcontra-continuous functions, International Journal of Mathematics and Mathematical Sciences 21 (1998), no. 1, 19-24.

[4] G. Balasubramanian, On some generalizations of compact spaces, Glasnik Matematički. Serija III 17(37) (1982), no. 2, 367-380. 
[5] F. Cammaroto and G. Santoro, Some counterexamples and properties on generalizations of Lindelöf spaces, International Journal of Mathematics and Mathematical Sciences 19 (1996), no. 4, 737746.

[6] J. Dontchev, Contra-continuous functions and strongly S-closed spaces, International Journal of Mathematics and Mathematical Sciences 19 (1996), no. 2, 303-310.

[7] J. Dontchev and M. Przemski, On the various decompositions of continuous and some weakly continuous functions, Acta Mathematica Hungarica 71 (1996), no. 1-2, 109-120.

[8] A. J. Fawakhreh and A. Kılıçman, Mappings and some decompositions of continuity on nearly Lindelöf spaces, Acta Mathematica Hungarica 97 (2002), no. 3, 199-206.

[9] S. V. Fomin, Extensions of topological spaces, Annals of Mathematics. Second Series 44 (1943), 471-480.

[10] H. O. Kim, Notes on C-compact spaces and functionally compact spaces, Kyungpook Mathematical Journal 10 (1970), 75-80.

[11] P. E. Long and L. L. Herrington, Strongly $\theta$-continuous functions, Journal of the Korean Mathematical Society 18 (1981), no. 1, 21-28.

[12] A. S. Mashhour, M. E. Abd El-Monsef, and S. N. El-Deep, On precontinuous and weak precontinuous mappings, Proceedings of the Mathematical and Physical Society of Egypt 53 (1982), 47-53 (1983).

[13] A. A. Nasef and T. Noiri, Some weak forms of almost continuity, Acta Mathematica Hungarica 74 (1997), no. 3, 211-219.

[14] T. Noiri, On $\delta$-continuous functions, Journal of the Korean Mathematical Society 16 (1980), no. $2,161-166$.

[15] J. H. Park and H. Y. Ha, A note on weakly quasi-continuous functions, International Journal of Mathematics and Mathematical Sciences 19 (1996), no. 4, 767-772.

[16] V. Popa and C. Stan, On a decomposition of quasi-continuity in topological spaces, Studii şi Cercetări Matematice 25 (1973), 41-43 (Romanian).

[17] M. K. Singal and S. Prabha Arya, On almost-regular spaces, Glasnik Matematički. Serija III 4 (24) (1969), 89-99.

[18] _ On nearly paracompact spaces, Matematički Vesnik 6 (21) (1969), 3-16.

[19] M. K. Singal and A. R. Singal, Almost-continuous mappings, Yokohama Mathematical Journal 16 (1968), 63-73.

[20] Mildly normal spaces, Kyungpook Mathematical Journal 13 (1973), 27-31.

[21] S. Willard and U. N. B. Dissanayake, The almost Lindelöf degree, Canadian Mathematical Bulletin 27 (1984), no. 4, 452-455.

A. J. Fawakhreh: Department of Mathematics, Philadelphia University, P.O. Box 1, Amman 19392, Jordan

E-mail address: afawakhreh@philadelphia.edu.jo

A. Kılıçman: Department of Mathematics, University Putra Malaysia, 43400 Serdang,

Selangor, Malaysia

E-mail address: akilic@fsas.upm.edu.my 


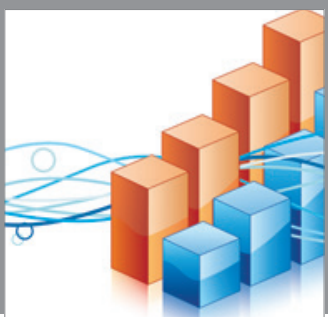

Advances in

Operations Research

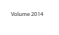

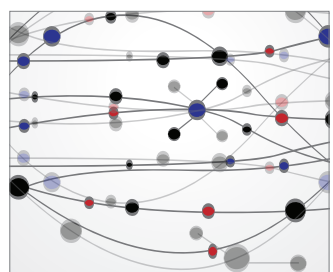

\section{The Scientific} World Journal
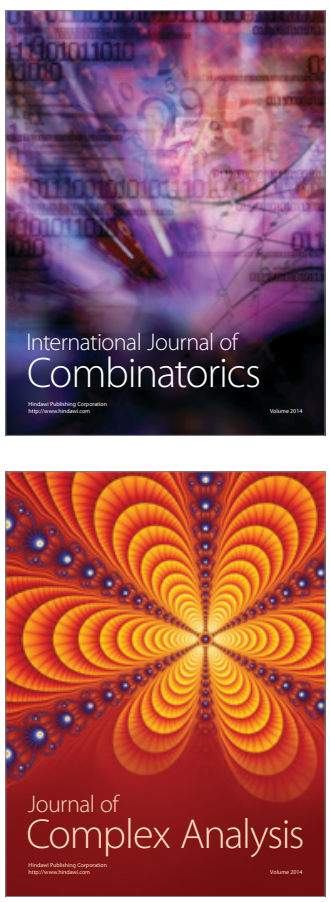

International Journal of

Mathematics and

Mathematical

Sciences
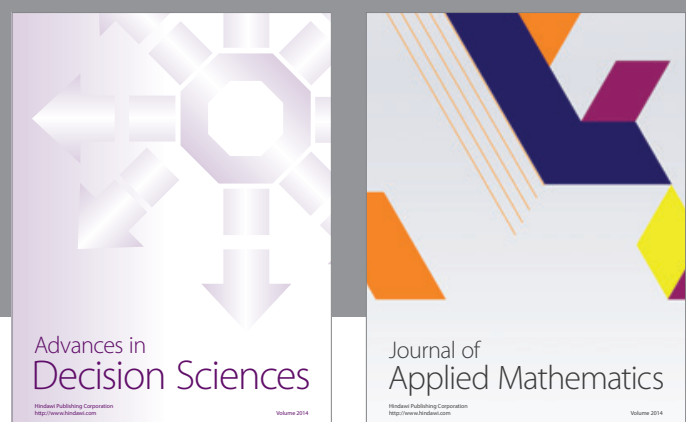

Journal of

Applied Mathematics
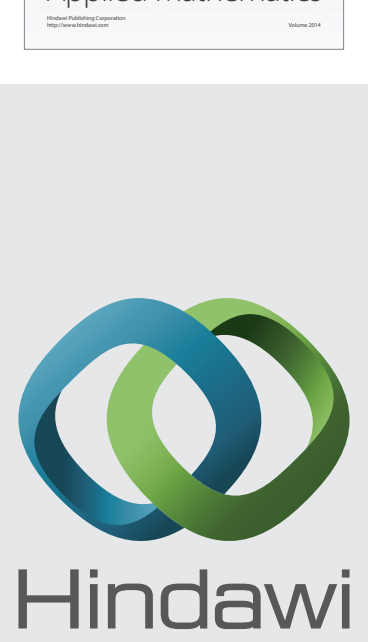

Submit your manuscripts at http://www.hindawi.com
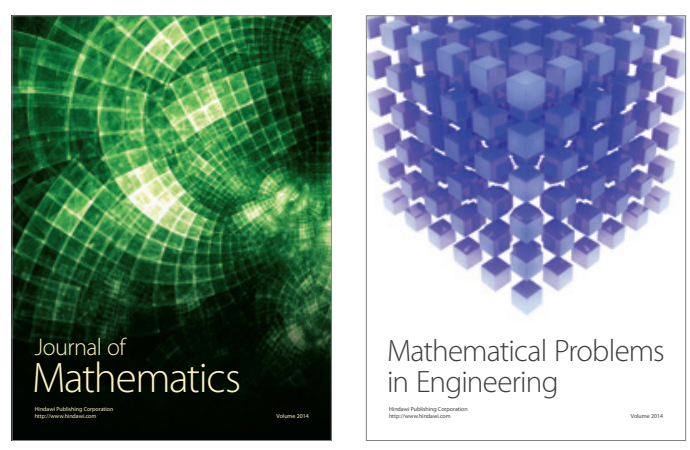

Mathematical Problems in Engineering
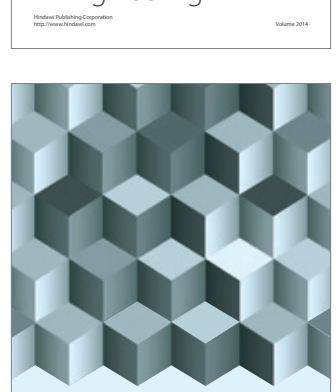

Journal of

Function Spaces
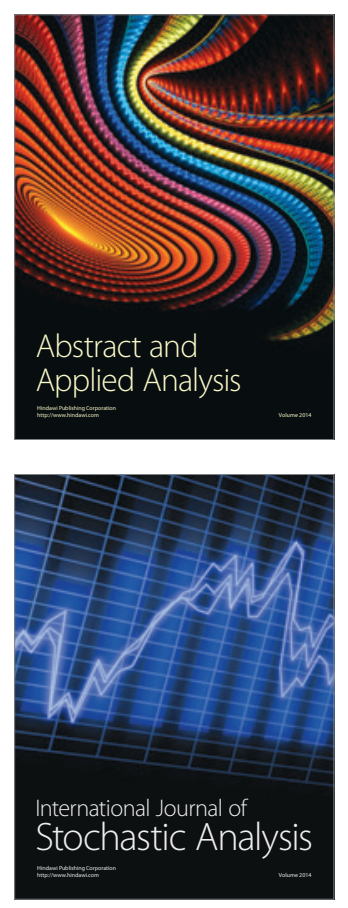

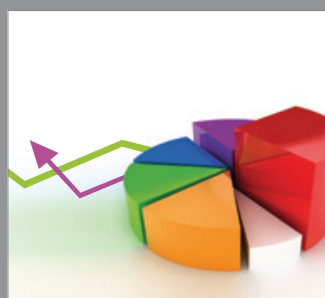

ournal of

Probability and Statistics

Promensencen
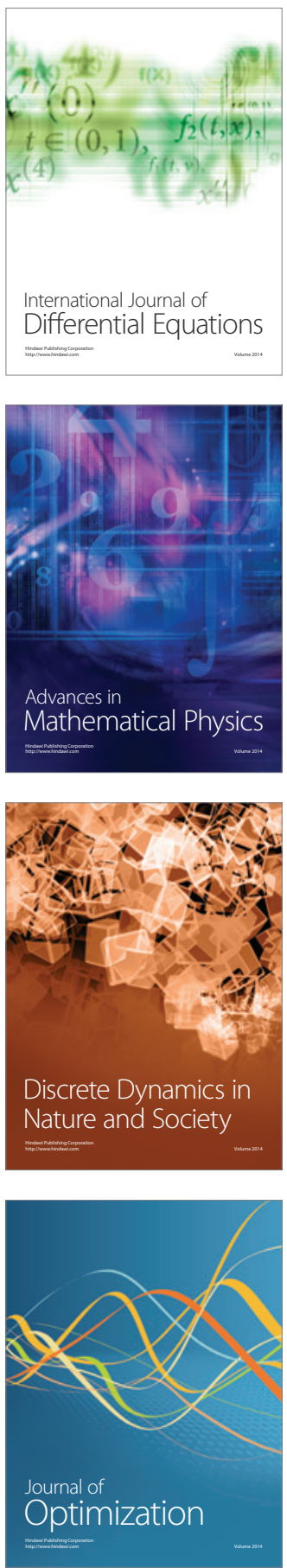